Ger J Exerc Sport Res 2021 · 51:135-145 https://doi.org/10.1007/s12662-020-00699-y Received: 3 September 2020

Accepted: 8 December 2020

Published online: 5 January 2021

(c) The Author(s) 2020

\author{
Hans-Erik Scharfen ${ }^{1,2}$ (D) Daniel Memmert ${ }^{2}$ \\ ${ }^{1}$ Neurocognition and Performance Lab, SV Werder Bremen, Franz-Böhmert-Str. 1, 28205 Bremen, \\ Germany \\ ${ }^{2}$ German Sport University Cologne, Cologne, Germany
}

\title{
Cognitive training in elite soccer players: evidence of narrow, but not broad transfer to visual and executive function
}

2012; Vestberg, Reinebo, Maurex, Ingvar, \& Petrovic, 2017; Verburgh, Scherder, Van Lange, \& Oosterlaan, 2014, 2016; Huijgen et al., 2015). These executive functions include the cognitive processes that regulate thoughts and actions, especially in nonroutine situations (Miyake \& Friedman, 2012). Additionally, they are further subdivided into core executive functions, which include working memory, cognitive flexibility and inhibitory control, and higher-level executive functions, involving reasoning, problemsolving and planning (Diamond, 2013). These higher-level functions altogether are also called metacognition (Vestberg et al., 2012).

Moreover, athletes with sophisticated domain-general visual functions like visual clarity and depth perception seem to have a higher success rate in team sports (Burris, Liu, \& Appelbaum, 2019; Roberts, Strudwick, \& Bennett, 2017). Although domain-general visual and executive functions are deeply linked to each other, their dissociation is quite essential in this case. The core area of vision provides sensory information of the outside world and depends on afferences (i.e. input streaming to the brain) for the most part. In contrast, executive functions are also linked to the processing of that sensory information (Gilbert \& Burgess, 2008).

Another line of evidence regarding these functions indicates that physical activity is a crucial booster for cognitive processes by triggering processes like enhanced cerebrovasculation and the release of neurotropic factors like BDNF (brain-derived neurotrophic factor; for review see Prakash, Voss, Erickson, \& Kramer, 2015; Cox et al., 2016). The combination of this physical boosting with the cognitive superiority of elite soccer players leads to the conclusion that extensive soccer practice might result in an implicit training of these functions. However, the mainly cross-sectional nature of this evidence does not allow causal conclusions.

Based on the superiority of elite athletes in terms of visual and executive functions, the interest to evaluate training possibilities of these skills is very high. One example of these training possibilities belongs to the cognitive component skill approach which targets fundamental subprocesses (for review see Appelbaum \& Erickson, 2018; Hadlow, Panchuk, Mann, Portus, \& Abernethy, 2018). A widely applied training-program associated with this approach is called NeuroTracker ${ }^{\mathrm{TM}}$ (NT) 3 dimensional (3D) multiple-object tracking (MOT) (Faubert, 2013; CogniSens Athletics Inc., Université de Montréal).

First longitudinal studies using this NT 3D-MOT to train young athletes showed heterogeneous results. One of these studies was conducted by Parsons et al. (2016), who examined university students after 5 weeks of training by using a quantitative electroencephalogram and a battery of neuropsychological tests. They found enhanced attention, visual information processing speed, working terms of executive functions (Vestberg,
Gustafson, Maurex, Ingvar, \& Petrovic, 
memory and a higher amount of allocatable neural resources. However, the applied working memory test is questionable as the required cognitive functions are related to short-term memory for the most part, whereas the other tests are valid. In another study, Fleddermann, Heppe, and Zentgraf (2019) compared cognitive and sport-specific adaptions of elite volleyball athletes after 8 weeks of training with those of an active control group. More specifically, processing speed, memory span, working speed, sustained attention, and a volleyballspecific test were analyzed through several neuropsychological tests. Significant improvements were found in processing speed and sustained attention. However, these results need to be interpreted with caution as sport-specific motor actions were part of the NT 3D-MOT training as well. Romeas, Guldner, and Faubert (2016) showed improved passing decision-making accuracy in amateur soccer players after 5 weeks of training. They analyzed small-sided soccer games in a pre-post design with an active and a passive control group. Contrary to those results, Moen, Hrozanova, and Stiles (2018) found no significant improvements in executive functions in elite athletes from dynamic sports after 5 weeks of training. However, those results need to be interpreted with caution as the number of absolved sessions in this study was highly differing.

Furthermore, one core principle of the NT 3D-MOT used in the previous studies is the adaptability of task difficulty. Concerning this principle, two opposed theories have been proposed to explain individual differences in training-related performance gains (for review see Karbach \& Unger, 2014). First, the magnification account suggests that individuals who already perform on a high level will benefit most from cognitive training. According to this theory, high-performing individuals have more cognitive capacities to acquire new skills. Second, the compensation theory assumes that lowperforming individuals will benefit more from cognitive training as their room for improvement may be relatively large (Karbach \& Unger, 2014).
By reviewing the current literature on NT 3D-MOT training in young athletes, it is conspicuous that only basic cognitive mechanisms like processing speed are improved. In contrast, higher cognitive processes as executive functions are not enhanced by training except for attention. Furthermore, none of the few studies investigated the covariate physical activity which is a crucial booster for cognitive functions (Prakash et al., 2015; Cox et al., 2016). Therefore, the depicted improvements after NT 3D-MOT training could be inaccurate due to this influence. Further, it is unclear whether the few improvements in fundamental cognitive processes are originated in this area or whether this is based on adapted processing of visual skills. This uncertainty is based on the so-called transfer phenomenon which entails the two opposing theories of narrow and broad transfer (Furley \& Memmert, 2011). These theories were further categorized by Zentgraf, Heppe, and Fleddermann (2017) into task-specific (i.e. improvements in the trained task), near (i.e. similar cognitive task), further (i.e. other not related cognitive tasks) and far (i.e. transfer to competition). In the present investigation task-specific, near- and further-transfer effects are examined. The study with the largest further-transfer results used a sample of university students (Parsons et al., 2016). Learning curves have been shown to differ substantially among elite athletes and university students (Faubert, 2013). This suggests that a simple transfer from changes in students to changes in elite athletes is not suitable and should be interpreted with caution when seeking to apply such findings to world-class elite athletes directly. Consequently, it is not sure that the improvements are not merely an effect of those physical exercises. Furthermore, three of the four studies investigated effects after five training weeks which is a relatively short time. Therefore, it might only depict a very limited snapshot of possible training effects.

Due to this substantial methodological heterogeneity in recent literature, an analysis from a fundamental standpoint showed that the expected involvement of visual and executive functions in the NT
3D-MOT is relatively low (•Table 3 in the appendix). This expectation is based on the requirements of the task, which are very specific and do not contain other visual or cognitive elements besides the MOT skill. Contrary, several claims are made that the NT 3D-MOT enhances executive and some visual functions like the visual field, depth perception and attention (Faubert \& Sidebottom, 2012; Parsons et al., 2016). The mismatch of these claims is accompanied by the low theoretical probability of fulfilling them. When also taking the heterogeneous literature on NT 3D-MOT transfer into consideration a fundamental examination of this tool seems essential in order to rigorously evaluate its practical importance (Walton, Keegan, Martin, \& Hallock, 2018).

In order to clarify the recent literature gaps and by following current recommendations (Harris, Wilson, \& Vine, 2018; Walton et al., 2018), the present investigation is unique in several key aspects. Namely, the aim was to analyze the transfer effects of training intervention with the NT 3D-MOT on both visual and executive functions in a pre-post test design with a training and a control group. Accordingly, the training intervention was conducted with a strict single-task NT 3D-MOT in elite soccer players between 17 and 21 years of age over 10 weeks. The applied visual tests are used for measures of the transfer due to their fundamental role in the process of perception (Burris et al., 2019; Hüttermann, Memmert, \& Simons, 2014). The executive function tests are included based on their apparent importance in elite soccer (e.g. Vestberg et al., 2012, 2017; Verburgh et al., 2014, 2016; Huijgen et al., 2015). Additionally, the covariate physical activity was included to analyze possible influences on the outcome measures. Moreover, the prediction of NT 3D-MOT performance gains using pretest performance was analyzed as well. Specifically, the opposing magnification and compensation theories were examined, proposing an amplification of higher or compensation of lower baseline performance, respectively. 


\section{Methods}

\section{Participants}

A total of 29 elite soccer players from the talent development program of the youth academy of a professional German soccer club were recruited. They were further divided into a training $(n=16)$ and a passive control group $(n=13)$ by their coaches. The participants were males born between 1997 and $2003\left(M_{\text {age }}=18.77\right.$ years, $\left.S D_{\text {age }}=1.42\right)$. At the time of data collection, their teams were playing at the top level of their respective age group (U19 team) or the fourth-highest senior league (U23 team). Participants did not report any behavioral, learning, or medical condition that might influence cognitive abilities. Their physical fitness and educational level was homogenous and written informed consent was obtained from every participant before commencing the experiment. The study was carried out in accordance with the Helsinki Declaration of 1975 and was approved by the ethics committee of the German Sport University Cologne.

\section{Measures}

Visual tests. The NT 3D-MOT task with the NeuroTracker ${ }^{\mathrm{Tm}}$ Core Program by CogniSens Athletics Inc. from the University of Montreal was used for the training intervention as well for one pre- and posttest. This task was used as a manipulation analysis to ensure that a possible lack of transfer is not based on a lack of improvement in the trained task. The program was depicted on a wall via a video projector. NT 3D-MOT settings were the same as in Faubert (2013). During the session, eight yellow balls were presented, of which four changed their color for $1 \mathrm{~s}$ to orange. Participants were asked to memorize these balls. Then, that all balls moved randomly through the $3 \mathrm{D}$ domain with a specific velocity for $8 \mathrm{~s}$. After $8 \mathrm{~s}$, the spheres stopped moving, and the participants were asked to indicate the four "orange" balls (targets). Afterwards, participants received feedback, and the next trial started. One session lasted about $8 \mathrm{~min}$ and consisted of 20 trials. The dependent measure was the average speed threshold among all trials (for detailed information see Faubert, 2013).

Attention window was assessed with the Attention Window task by Hüttermann et al. (2014). The individual attention breadth on diagonal, horizontal and vertical axis was measured. During each trial, participants were instructed to fixate a central point and try to spot a white triangle within a circle $\left(1.1^{\circ}\right.$ diameters) among square distractors $\left(1.1^{\circ} \times 1.1^{\circ}\right)$. Across trials, the target appeared at varying distances from the fixation point $\left(10^{\circ}, 20^{\circ}\right.$, and $\left.30^{\circ}\right)$ along with one of eight equally spaced radial lines that originated from a square in the center of the display ( $45^{\circ}$ apart). This random display was flashed for $12 \mathrm{~ms}$ and followed by a colorful mask ( $100 \mathrm{~ms})$. After every mask, subjects were asked to indicate how many white triangles they had just seen in the different locations depending on the orientation of the items. Participants completed 144 trials. This particular task measures how well people can attend to objects appearing far from fixation. The high quality of the testing criteria has been described in a recent review (Hüttermann \& Memmert, 2017). The assessment lasted about $12 \mathrm{~min}$, and the dependent measure was the accumulated value of all three dimensions (diagonal, horizontal, vertical) divided by the number of the measurements (i.e. three) in degree (Scharfen \& Memmert, 2019b).

The Senaptec Sensory Station was used to assess a variety of visual skills (i.e. visual clarity, contrast sensitivity, depth perception, near-far quickness, target capture, perception span, multiple-object tracking, reaction time) with proven reliability (for a detailed description of the procedure and reliability see Erickson et al., 2011). The assessment lasted about 20 min. Dependent measures of the individual visual functions are described below:

- Visual clarity: ability to process nonmoving visual information while standing still. Dependent variable: threshold of the static visual acuity (depending on individual adapting staircase algorithm)
Ger J Exerc Sport Res 2021 · 51:135-145 https://doi.org/10.1007/s12662-020-00699-y (c) The Author(s) 2020

\section{H.-E. Scharfen · D. Memmert} Cognitive training in elite soccer players: evidence of narrow, but not broad transfer to visual and executive function

\section{Abstract}

Visual and executive functions have been suggested to be crucial in high-demanding team sports. Consequently, the interest in evaluating training possibilities of these functions is relatively high. However, easily applicable training tools, as well as evidence of their efficacy, especially in the present group of age (i.e. 17-21 years) and performance level, are scarce. Therefore, the present study aimed to evaluate the effectiveness and transfer of an essential cognitive training tool (i.e. NeuroTracker [NT] three dimensional [3D] multipleobject tracking [MOT]) in youth elite soccer players. Visual and executive functions were analyzed in a pre-post test design with an intervention and a control group after 10 weeks of training twice a week. Physical activity was included as a possible covariate. Results show meaningful benefits in the trained ability (i.e. MOT) besides small but negligible improvements in visual clarity and inhibition for the intervention group. Consequently, strict single-task NT 3D-MOT seems to have little transfer to other visual or executive functions. However, future studies should investigate the effects of sportspecific dual-task NT 3D-MOT to analyze possible multitasking adaptations further.

Keywords

Perceptual functions - Multiple-object tracking $\cdot$ High-performance athletes $\cdot$ Brain training $\cdot$ Soccer

- Contrast sensitivity: ability to process spatial or temporal information about objects and their backgrounds under varying lighting conditions. Dependent variable: threshold sensitivity (depending on individual adapting staircase algorithm)

- Depth perception: determining distance and spatial localization of an object. Dependent variable: threshold depth perception (depending on individual adapting staircase algorithm) 
- Near-far quickness: ability to quickly switch the gaze between far, intermediate, and near distances requiring rapid accommodative-vergence responses. Dependent variable: number of correct responses

- Target capture: ability to process moving visual information while standing still. Dependent variable: threshold stimulus exposure (depending on individual adapting staircase algorithm)

- Perception span: speed and span of recognition of tachistoscopic information. Dependent variable: cumulative number of correct responses

- Reaction time: reacting as fast as possible to occurring visual stimuli by touching them. Dependent variable: elapsed time between onset and touching of the test stimuli

Executive function tests. Core executive function tests

Working memory capacity was measured by using the well-established working memory span test by Conway et al. (2005). It measures the athlete's ability to direct attention toward the current task without getting distracted by other thoughts. More specifically, we used a counting span task (see Kane et al., 2004 for a detailed description), as the simplicity of this processing task makes it usable for almost any type of participant (Conway et al., 2005). The instructions were presented as a written text on the computer screen. The counting span task involved counting specific shapes among distractors and then remembering the count totals for later recall. Each stimulus display contained randomly arranged dark blue circles, green circles, and dark blue squares. The task of the participants was to count aloud the dark blue circles and then name the total count aloud at the end. A recall mask occurred after 2-6 stimulus displays into which participants had to fill their memorized count totals in the exact order they had been displayed in. The participants counting span score was a partial credit load score (cf. Conway et al., 2005) which represents the sum of all correctly identified elements-whereby a correctly recalled item from a set containing two items re- ceives 2 points, and a correctly recalled item from a set with 6 items receives 6 points-divided by the maximum possible score. Good reliability and validity for this test have been reported elsewhere (see Conway et al., 2005). The test consisted of 15 trials, and the assessment lasted about $13 \mathrm{~min}$. The dependent measure was the score of correctly memorized objects in percentage from a maximum of 100 (Scharfen \& Memmert, 2019b).

Cognitive flexibility was measured with the Trail Making Test (TMT) which consisted of two parts (A and B) (Sánchez-Cubillo et al., 2009). The TMT-A is regularly applied to assess visuoperceptual abilities, whereas TMT-B is used to assess cognitive flexibility (Crowe, 1998). A smaller B-A difference suggests better cognitive flexibility (for detailed a description see Huijgen et al., 2015). A validated tablet version of the TMT was used which is congruent with the traditional pen-paper version that has been shown to be reliable and valid (Sánchez-Cubillo et al., 2009; Delbaere \& Lord, 2015). The assessment lasted about $5 \mathrm{~min}$, and the dependent measure was the B-A difference in seconds.

Inhibition was measured with a GoNoGo task which was also included in the Senaptec Sensory Station test battery. Yellow-green dots required the athlete to touch them as fast as possible (i.e. Go condition) whereas red dots should not be touched (i.e. NoGo condition). Ninetysix total dots (64 yellow-green, 32 red) were presented in a pseudorandomized sequence to maintain equivalent spatial distribution. The assessment lasted about $3 \mathrm{~min}$, and the dependent measure was the cumulative value of touched Go stimuli minus any NoGo stimuli touched (for a detailed description see Erickson et al., 2011).

\section{Higher-level executive function test.} Metacognition was measured with the standardized Design Fluency test, which assesses online multiprocessing such as creativity, response inhibition, and cognitive flexibility (Homack, Lee, \& Riccio, 2005; Swanson, 2005). This test belongs to the Delis-Kaplan system and assesses performance relying on both core- and higher-level executive func- tions, thus stimulating the executive chain of decision-making. The task requires connecting dots with four lines under time pressure $(60 \mathrm{~s})$ to produce as many different patterns as possible. The participant is not allowed to use the same solution twice. A computerized version of this test with good reliability was used. The assessment lasted about 5 min (i.e. 4 rounds of $60 \mathrm{~s}$ ), and the dependent measure was the number of unique created patterns (for a detailed description see Woods, Wyma, Herron, \& Yund, 2016).

\section{Procedure}

Visual and executive function test data were collected in a separate and quiet room. The test session consisted of one session lasting approximately $75 \mathrm{~min}$ and was conducted before a soccer training. A battery of six tasks described above was used to explore individual differences in visual and executive functions. The order of the tests was fixed for all participants, which is a standard method in neuropsychological assessment, especially with small samples: 1) NT 3DMOT, 2) Attention Window, 3) Working Memory Capacity, 4) Metacognition, 5) Cognitive Flexibility, 6) Visual Functions and 7) Inhibition. In the assessments 1, 2, 3, 4 and 5 all participants were instructed to sit in a comfortable position leaning against the backrest of the chair so that the distance to the screen was the same for all the players. Further, a computer screen was used for all these assessments (i.e. 1-5) except for $1)$ in which the program was depicted on a wall via a video projector. Test 6) was conducted by utilizing the Senaptec Sensory Station with the player standing in front of it (for a detailed description of the procedure see Erickson et al., 2011). The NT 3D-MOT was used for the training intervention in the training group besides their regular team practice, whereas the control group continued their regular team practice without any additional tasks. One experimenter tested all players in a standardized process. Data on physical activity included the training duration per week per player as daily documented by the coaches of 


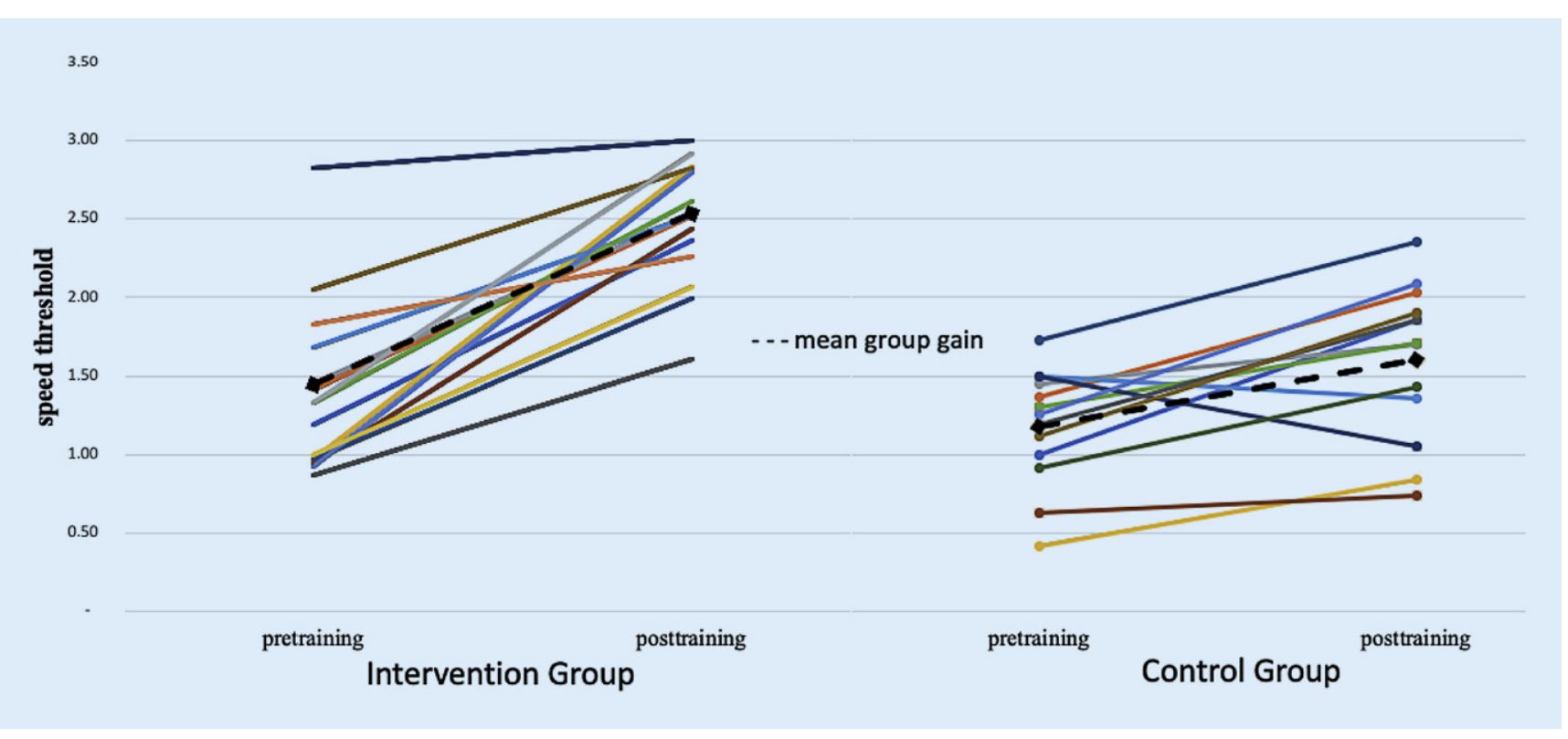

Fig. $1 \Delta$ Mean speed thresholds of the NT 3D-MOT of all athletes are represented in continuous lines, whereas dashed lines represent mean speed threshold gains

the individual teams, in which only the team training activities were considered. These data were further combined with the leisure time activity collected through a questionnaire (i.e. asking the player to state their average leisure time activity in hours per week including all physical activity besides the regular training program of the youth academy).

\section{Training intervention}

The NT 3D-MOT task (described above, see also - Table 3 in the appendix) was used for the training intervention. The training group $\left(n=16 ; M_{\text {age }}=18.87\right.$ years; $S D=0.89$ ) actively practiced 20 times; twice a week for 10 weeks in addition to the regular ball and athletic training. During each practice, they participated in three CORE sessions of NT, 3D-MOT (Romeas et al., 2016). Every participant reached a total of 60 sessions at the end of the training phase. They followed the same standard procedure and completed the first five practices seated and the following fifteen practices standing (i.e. 15 and 45 sessions; respectively). For a more detailed description of the NT 3D-MOT see Romeas et al. (2016). The control group $\left(n=13 ; M_{\text {age }}=18.64\right.$ years; $S D=1.94)$ progressed with their regular practice, such as ball and athletic prac- tice. Physical activity (i.e. training time and physical activity in leisure activity) was controlled for in both groups as it has been shown to improve cognitive performance (Prakash et al., 2015; Cox et al., 2016).

\section{Statistical analysis}

Data were analyzed using IBM SPSS Statistics 26.0.0 (Armonk, NY, USA). Instead of conducting null-hypothesis significance tests, we followed recent recommendations to focus on estimation for best reporting and analysis practice (Cumming, 2012, 2014); we report effect-sizes with $95 \%$ confidence intervals which have also been conducted successfully elsewhere (e.g. see Kreitz, Furley, Memmert, \& Simons, 2014; Ivarsson, Andersen, Johnson, \& Lindwall, 2013). Effect sizes (Cohen's $d$ ) of 0.2, 0.5, and 0.8 represent small, medium and large effect size estimates (Cohen, 1988). Visual inference in conjunction to the analysis of proportion overlap was conducted in order to compare the mean performance change of both groups directly (i.e. the smaller the overlap of the confidence intervals the larger the meaningful difference). This procedure has been proposed as the best reporting practice (Cumming \& Finch, 2005).
With regard to testing predictive ability of pretest performance on NT 3DMOT performance gains and to analyze the two opposed theories, we examined the correlation of pretest performance (i.e. executive and visual functions) and performance gains in the NT 3D-MOT. Previously, NT 3D-MOT performance gains were calculated by analyzing the difference from pre- to posttest performance using Microsoft Excel Version 16.10 (2016). Shapiro-Wilk test was used for testing for normal distributions. Not all variables were normally distributed, as assessed by Shapiro-Wilk's test $(p<0.05)$. Therefore, the Spearman's correlation coefficient test was used to investigate the correlation between the player's pretest performance and performance gains in the NT 3D-MOT.

\section{Results}

Descriptive statistics, as well as confidence intervals and effect sizes of pre- and posttests of both groups' visual and executive functions are depicted in • Table 1. The intragroup performance differences (i.e. among pre- and posttest of the individual groups) in the NT 3D-MOT are presented in - Fig. 1. The intraintervention group effect size difference of performance change in NT 3D-MOT, MOT, 


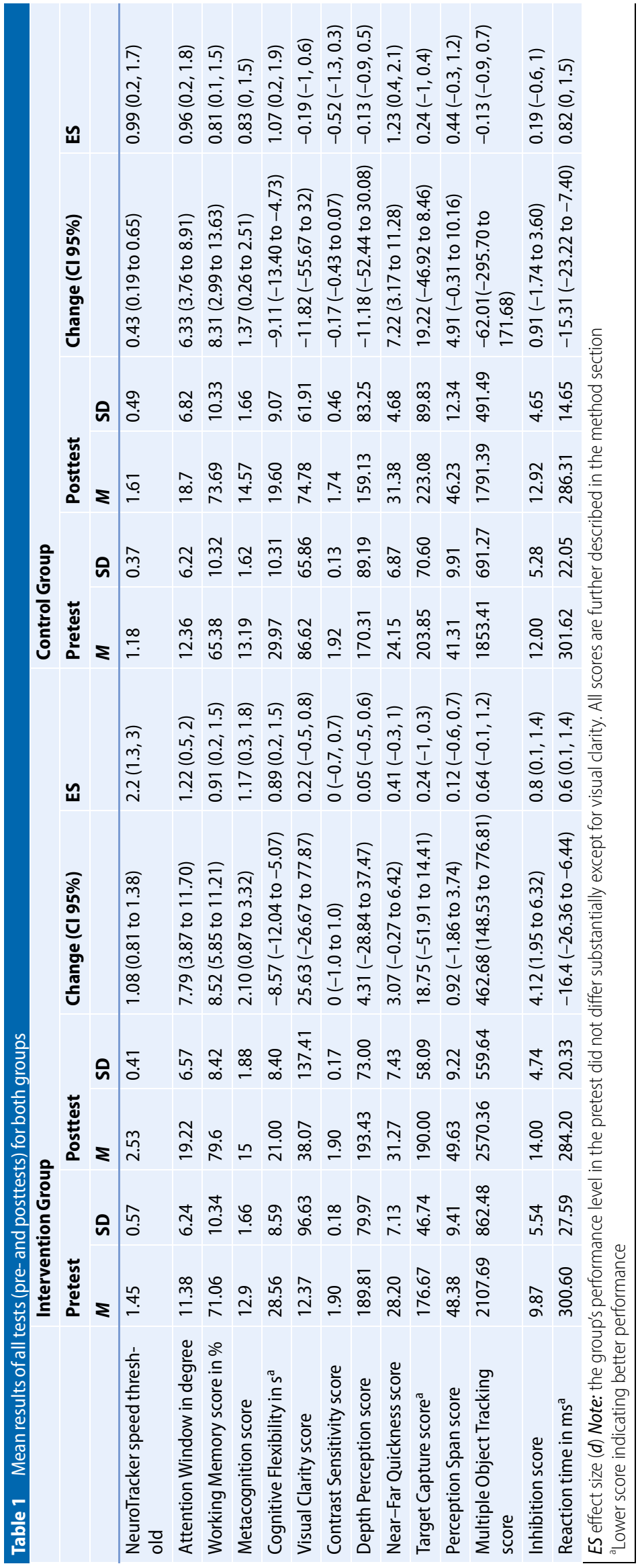

and inhibition was more extensive (i.e. medium to large) than the effect size difference of performance change in the control group (i.e. large, negligible and small). Contrary, the intragroup performance differences for near-far quickness was larger in the control group than in the intervention group. However, the proportion overlap (i.e. small or not existent indicating meaningful difference, Cumming \& Finch, 2005) of the intergroup effect size difference of performance change (i.e. pre-post changes from the intervention compared to the control group) was only meaningful in the NT 3D-MOT- and MOT score as depicted in $\bullet$ Fig. 2.

Confidence intervals and effect sizes of the results showed medium to large intra-intervention group improvements in the performance of NT 3D-MOT, working memory, cognitive flexibility, inhibition, metacognition, MOT, attention window and processing speed (i.e. reaction time) besides a large effect size in near-far quickness performance in the control group. All other parameters demonstrate no or only small changes which could also be based on random fluctuations.

\section{Prediction of NT 3D-MOT performance gains}

NT 3D-MOT performance was strongly negatively correlated with NT 3D-MOT performance gains $\left[\mathrm{r}_{\mathrm{s}}(16)=-0.851\right.$ (CI: $-1.5,-0.3), d=3.23$ ], whereas target capture was strongly positively correlated with NT 3D-MOT performance gains $\left[\mathrm{r}_{\mathrm{s}}(16)=0.655\right.$, (CI: $\left.\left.-0.1,1\right), d=1.72\right]$. No other correlation appeared to be substantially meaningful (- Table 2 in the appendix).

\section{Physical activity}

The total time of physical activity for the duration of the study was $84.22 \mathrm{~h}$ ( $S D$ : 25.17) for the intervention group and $76.44 \mathrm{~h}$ (SD: 26.05) for the control group. The effect of the difference between the physical activity of both groups was small to medium $d=-0.3$ (CI: $-1,0.4$ ). 


\section{Discussion}

The current study addressed the question of whether a 10-week training program with the NT 3D-MOT evokes transfer effects to visual and executive functions in elite soccer players. The absence of any meaningful changes indicates a lack of further-transfer to other not directly trained visual and executive functions except for task-specific effects and neartransfer to skills of NT 3D-MOT and MOT. The previous comparison of visual and executive functions and their theoretical requirement in NT 3D-MOT did not indicate any common ground (- Table 3 in the Appendix), which seems to explain the lack of meaningful furthertransfer. Moreover, this lack also appears to be based on the specificity of the training task which includes some aspects of dynamic soccer situations but does not cover the perception and action combinations of these situations (Romeas, Chaumillon, Labbé, \& Faubert, 2019). Thus, it could be argued that this dearth of several broad perception and action combinations may be one of the reasons for the absence of any further-transfer which is in line with a current review (Hadlow et al., 2018).

This lack of further-transfer to executive functions is in line with previous studies of Moen et al. (2018) and Fleddermann et al. (2019). However, increases in sustained attention and processing speed were found in the latter study. The investigation of Parsons et al. (2016) is in contrast to the current findings as improvements in several cognitive functions were found. Though, the task-specific effects and near-transfer to the NT 3D-MOT and MOT are in line with previous studies of Fleddermann et al. (2019) and Faubert (2013) which demonstrate that the athletes have an extraordinary capacity for learning an unpredictable and complex visual tracking task (Faubert, 2013). Moreover, this practice improvement in NT 3D-MOT and MOT underlines that cognitive functions are trainable from a fundamental standpoint although this malleability may only be achievable in terms of neartransfer training effects (Bryck \& Fisher, 2012; Fields, 2015). Consequently, future studies should integrate brain-scanning tools to investigate functional or structural adaptations of the brain like more efficient neural processing as these are the building stones of performance improvements.

Overall, it seems to be the case that the NT 3D-MOT only evokes task-specific practice effects with little near- and no further-transfer to other visual and executive functions which is in line with a previous review (Diamond \& Ling, 2016). Further, it might be stated that the improvements of NT 3D-MOT and its transfer to soccer-specific decision making (Romeas et al., 2016) are mainly originated in the task-specific MOT enhancement and not due to changes of visual abilities or cognitive processing. This lack of further-transfer substantially challenges the statement that the NT 3D-MOT is a "Gold Standard Cognitive Enhancer" (Parsons et al., 2016) which enhances executive and some visual functions as the visual field, depth perception and attention (Faubert \& Sidebottom, 2012; Parsons et al., 2016). Additionally, the absence of further-transfer confirms the vast amount of studies showing that executive functions must be targeted specifically in training in order to improve them (for review see Diamond \& Ling, 2016).

Nevertheless, even the MOT ability alone seems to be crucial for dynamic sports (Mangine et al., 2014; for a metaanalysis see Scharfen \& Memmert, 2019a) and its training is assumed to lead to aforementioned soccer-specific decisionmaking improvements at least in amateur athletes (Romeas et al., 2016). However, it needs to be taken into consideration that this type of strict single-task NT 3D-MOT practice only depicts the first step in the training progression plan of the NT 3D-MOT. The next steps after an initial familiarization phase in sitting and standing positions would be balancing and dual-tasking with sports-specific movements. In terms of future practical application, it is highly interesting if those further progression steps yield larger further- or even far-transfer effects. A first dual-task study indicates the general superiority of dual-compared to single tasktraining with the NT 3D-MOT (Romeas et al., 2019).
Furthermore, elite athletes are already performing on a high cognitive level. Therefore, even small improvements like those found in the current investigation could be a meaningful change in a real game situation (see Change in $\bullet$ Table 1 ). This high cognitive performance level which is mostly present in cross-sectional studies of elite soccer players seems to be advantageous regardless of whether this is based on a matter of selection or implicit training (i.e. nature vs nurture).

The covariate physical activity differed only with a small to medium effect size which suggests that changes may be based on the cognitive training for the most part. Further, the second rationale of the study addressed the controversy of the two opposing theories aiming to explain individual differences in training-related performance gains (i.e. magnification vs compensation theory; Karbach \& Unger, 2014). The strong negative correlation of NT 3D-MOT performance, perception span and target capture (reversed performance scale-depicting a strong positive correlation) in the pretest and NT 3D-MOT performance gains may favor the compensation theory. This theory assumes that individuals with lower baseline performance have a more considerable gain potential from cognitive training as their room for improvement may be relatively large.

Concerning the practical applications, it can be stated that the NT 3D-MOT is a useful tool to enhance the athlete's multiple-object tracking skill which alone seems to be crucial for dynamic sports (Mangine et al., 2014; Scharfen \& Memmert, 2019a). Nevertheless, the present findings imply that the single-task NT 3D-MOT training is not suitable for enhancing other visual or executive functions. Future studies are required to examine the possible transfer effects of a dual-task training mode. As the absence of further transfer adds to the vast amount of previous studies emphasizing the need to target executive functions to improve them directly it is necessary to include specific elements of these executive functions in the training regime. Specifically, the integration of these elements in on- and off-pitch training could intensify the effects of the natural advan- 

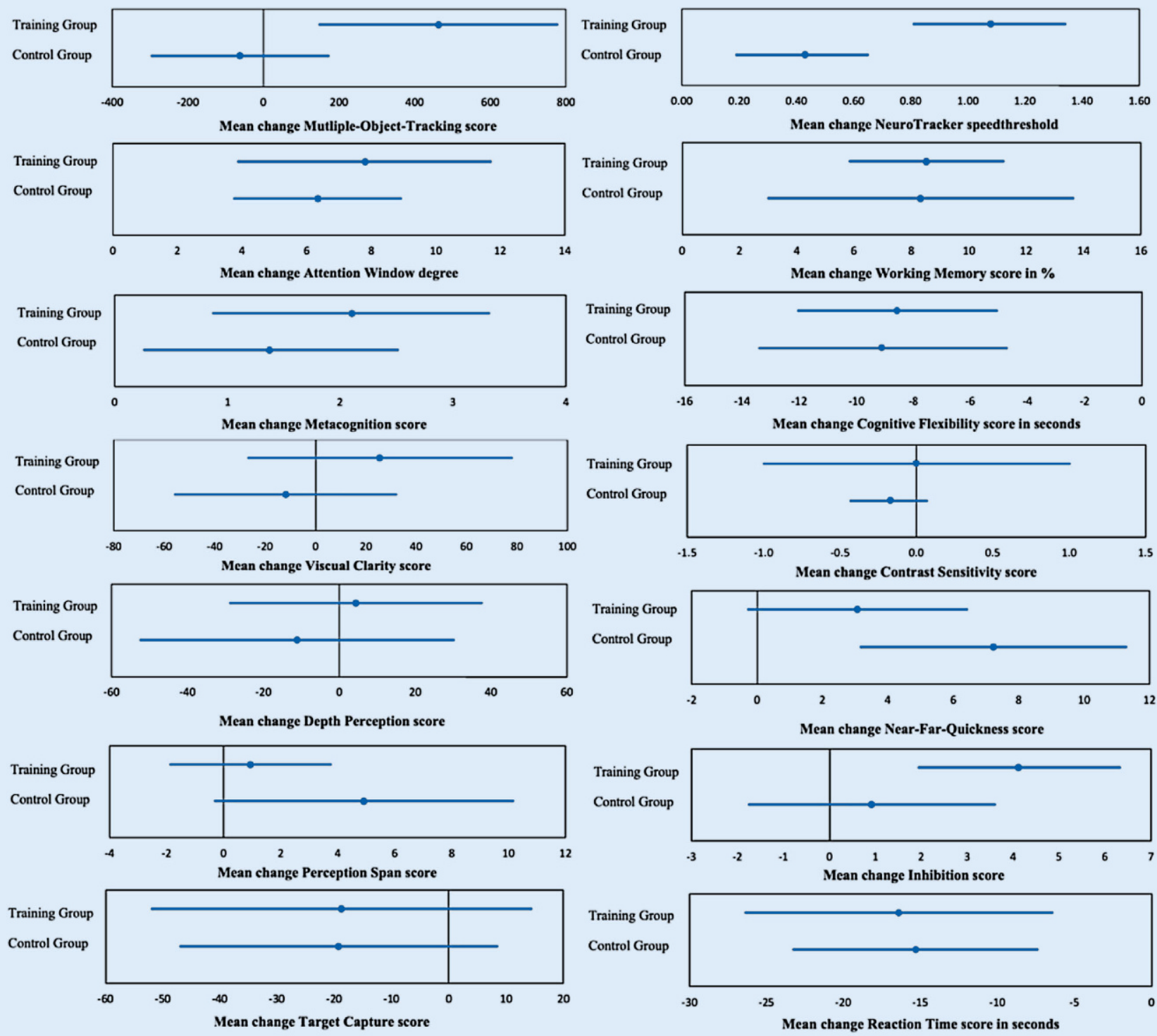

Fig. $2 \Delta$ Mean performance changes with $95 \%$ confidence intervals $(\mathrm{Cl})$ of all tests for both groups

tage/selection of players with superior executive functions or the implicit training of them by playing soccer as observed in elite players. Thus, future studies investigating sport-specific cognitive training, specifically targeting certain elements of executive functions either on- or off-pitch are highly relevant as the field of cognitive performance enhancement in eliteathletic populations is still in its infancy. Further, the NT 3D-MOT seems to be a helpful training tool to improve the multiple-object tracking skill, especially of players which have a deficit in this domain.
Some limitations of the present study also need to be acknowledged. In general, the sample size is not big enough to draw final inferences which can be seen in the relative breadth of the $95 \%$ confidence intervals. Based on these findings, future studies should replicate this with a larger sample and with a second intervention phase, including dual-task NT 3D-MOT training. Furthermore, it needs to be taken into consideration that other possible adaptions of the athlete's brain cannot be ruled out (e.g. higher degree of myelination, more efficient energy usage) as only the performance output of the brain but not the underlying processing mech- anisms had been tested. Karbacher and Unger (2014) proposed such tools and the study of Parsons et al. (2016) found altered brainwaves as a function of the NT 3D-MOT training which matches current findings of activity-dependent plasticity in the brain (Fields, 2015). Moreover, although the covariate physical activity was analyzed, other factors like some players being youth national players were still present, which could have influenced the outcome. 


\section{Conclusion}

Studies on the effectiveness and transfer of lab-based cognitive training programs in elite sports are scarce. Therefore, this study is one of only a few investigating transfers to a broad variety of visual and executive functions. Generally, results hint at the plasticity of these functions and their improvement as a function of additional lab-based training. The outcomes showed meaningful near-transfer benefits to the trained ability (i.e. MOT) besides minor improvements in a few other tasks (i.e. inhibition, visual clarity). Nevertheless, none of the other parameters showed meaningful further-transfer improvements or no improvements at all in the intervention group compared to the control group. The results may reduce the possible areas of further-transfer of the NT 3D-MOT and underpins the necessity of brain-scanning tools in future studies examining training effects of visual and executive functions. Investigations of transfer effects of NT 3DMOT dual-task training are needed to further rule out its field of application for practitioners.

\section{Corresponding address}

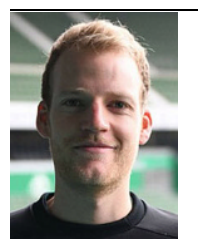

\section{Hans-Erik Scharfen}

German Sport University

Cologne

Am Sportpark Müngersdorf 6 50933 Cologne, Germany h.e.scharfen@gmx.de

Funding. This work was supported by SV Werder Bremen GmbH \& Co KGaA.

Funding. Open Access funding enabled and organized by Projekt DEAL.

\section{Compliance with ethical guidelines}

Conflict of interest. H.-E. Scharfen and D. Memmert declare that they have no competing interests.

For this article no studies with were performed by any of the authors. All studies performed were in accordance with the ethical standards indicated in each case.

Open Access. This article is licensed under a Creative Commons Attribution 4.0 International License, which permits use, sharing, adaptation, distribution and re- production in any medium or format, as long as you give appropriate credit to the original author(s) and the source, provide a link to the Creative Commons licence, and indicate if changes were made. The images or other third party material in this article are included in the article's Creative Commons licence, unless indicated otherwise in a credit line to the material. If material is not included in the article's Creative Commons licence and your intended use is not permitted by statutory regulation or exceeds the permitted use, you will need to obtain permission directly from the copyright holder. To view a copy of this licence, visit http://creativecommons.org/licenses/by/4.0/.

\section{Appendix}

Table 2 Correlations $\mathrm{r}_{\mathrm{s}}$ between pretest performance (i.e. executive and visual functions) and NT 3D-MOT performance gains

\begin{tabular}{|c|c|c|}
\hline & & NT Gain \\
\hline \multirow[t]{2}{*}{ NT 3D-MOT } & Correlation coefficient & -0.851 \\
\hline & $\mathrm{Cl}$ & $-1.5,-0.3$ \\
\hline \multirow[t]{2}{*}{ Attention Window } & Correlation coefficient & -0.287 \\
\hline & $\mathrm{Cl}$ & $-0.8,0.6$ \\
\hline \multirow[t]{2}{*}{ Working Memory } & Correlation coefficient & -0.286 \\
\hline & $\mathrm{Cl}$ & $-0.5,0.6$ \\
\hline \multirow[t]{2}{*}{ Metacognition } & Correlation coefficient & -0.373 \\
\hline & $\mathrm{Cl}$ & $-0.5,0.46$ \\
\hline \multirow[t]{2}{*}{ Cognitive Flexibility } & Correlation coefficient & 0.021 \\
\hline & $\mathrm{Cl}$ & $-1.1,0.2$ \\
\hline \multirow[t]{2}{*}{ Visual Clarity } & Correlation coefficient & -0.180 \\
\hline & $\mathrm{Cl}$ & $-0.5,0.5$ \\
\hline \multirow[t]{2}{*}{ Contrast Sensitivity } & Correlation coefficient & 0.111 \\
\hline & $\mathrm{Cl}$ & $-0.37,0.6$ \\
\hline \multirow[t]{2}{*}{ Depth Perception } & Correlation coefficient & 0.234 \\
\hline & $\mathrm{Cl}$ & $-0.7,0.12$ \\
\hline \multirow[t]{2}{*}{ Near-Far Quickness } & Correlation coefficient & 0.108 \\
\hline & $\mathrm{Cl}$ & $-0.4,1.4$ \\
\hline \multirow[t]{2}{*}{ Target Capture } & Correlation coefficient & 0.655 \\
\hline & $\mathrm{Cl}$ & $0.12,1.1$ \\
\hline \multirow[t]{2}{*}{ Perception Span } & Correlation coefficient & -0.164 \\
\hline & $\mathrm{Cl}$ & $-1.5,-0.05$ \\
\hline \multirow[t]{2}{*}{ Multiple-Object Tracking } & Correlation coefficient & -0.353 \\
\hline & $\mathrm{Cl}$ & $-0.73,0.15$ \\
\hline \multirow[t]{2}{*}{ Inhibition } & Correlation coefficient & 0.238 \\
\hline & $\mathrm{Cl}$ & $-0.185,1.2$ \\
\hline \multirow[t]{2}{*}{ Reaction } & Correlation coefficient & -0.028 \\
\hline & $\mathrm{Cl}$ & $-0.92,0.26$ \\
\hline
\end{tabular}


Table 3 Comparisons of definitions of executive and visual functions and requirements in the NT 3D-MOT(definitions based on 1) executive functions: Diamond [2013]; Miyake and Friedman [2012], 2) visual functions: Erickson et al., [2011])

Working Memory
(Short-Term Memory)
Cognitive Flexibility
Inhibition
Response Inhibition
Cognitive Inhibition
Selective or Focused Attention (Interference
control)

Metacognition

\section{Visual Function \\ Visual Clarity \\ Contrast Sensitivity}

Depth Perception

Near-Far Quickness

Target Capturing

Perception Span

Reaction time

Attention Window

\section{References}

Appelbaum, L. G., \& Erickson, G. (2018). Sports vision training: a review of the state-of-theart in digital training techniques. International Review of Sport and Exercise Psychology, 11(1), 160-189. https://doi.org/10.1080/1750984X. 2016.1266376.

Bryck, R. L., \& Fisher, P. A. (2012). Training the brain practical applications of neural plasticity from the intersection of cognitive neuroscience, developmental psychology, and prevention science. American Psychologist, 67(2), 87-100. https://doi.org/10.1037/a0024657.

Burris, K., Liu, S., \& Appelbaum, L. (2019). Visualmotor expertise in athletes: Insights from semiparametric modelling of 2317 athletes tested on the Nike SPARQ Sensory Station. Journal of Sports Sciences. https://doi.org/10. 1080/02640414.2019.1698090.

Callan, D.E., \& Naito, E. (2014). Neural processes distinguishing elite from expert and novice athletes. Cognitive and Behavioral Neurology, 27(4), 183-188. https://doi.org/10.1097/WNN. 0000000000000043.

Cohen, J. (1988). Statistical power analysis for the behavioral sciences (2nd edn.). Hillsdale: Erlbaum.

Conway, A. R. A., Kane, M. J., Bunting, M. F., Hambrick, D. Z., Wilhelm, O., \& Engle, R. W. (2005). Working

\section{Definition}

Holding information in mind and manipulating it just holding information in mind

Changing perspectives or approaches to a problem, flexibly adjusting to new demands, rules, or priorities (as in switching between tasks)

Deliberate overriding of dominant or prepotent responses Inhibition of thoughts/memories Inhibition at the level of attention

Requirement in NT 3D-MOT

Not required, no manipulation Eventually required

Not required, no changes

Not required, no overriding Eventually required Eventually required

The ability to reason, problem-solving, and to see patterns or Not required relations among items

Visual acuity for fine details at a distance

Visual system's ability to process spatial or temporal information about objects and their backgrounds under varying lighting conditions

Determining distance and spatial localization of an object

Ability of quick accommodative-vergence facility in binocular Not required, no quick near-far saccadic responses to images at near and far distances

Ability of the visual system to resolve detail when there is relative movement between the target and the observer Ability to remember and recreate visual patterns

Ability to react and respond to a simple visual stimulus

Individual attention breadth on diagonal, horizontal and vertical axis
Not required, no fine details

Not required, no varying lighting conditions

Not required, only tracking but not determining localizations at different distances changes

Not required, movement is present but no need to resolve in detail

Not required as the visual pattern is not static but constantly changing

Not required, no quick reaction required

Eventually required memory span tasks: a review and a user's guide Psychonomic Bulletin and Revie, 12,769-786.

Cox, E. P., O'Dwyer, N., Cook, R., Vetter, M., Cheng, H. L. Rooney, K., \& O'Connor, H. (2016). Relationship between physical activity and cognitive function in apparently healthy young to middle-aged adults: a systematic review. Journal of Science and Medicine in Sport, 19(8), 616-628. https:// doi.org/10.1016/j.jsams.2015.09.003.

Crowe, S.F. (1998). The differential contribution of mental tracking, cognitive flexibility, visual search, and motor speed to performance on parts $A$ and $B$ of the trail making test. Journal of Clinical Psychology, 54(5), 585-591. https://doi.org/10.1002/(sici)10974679(199808)54:5/585::aid-jclp4〉3.0.co;2-k.

Cumming, G. (2012). Understanding the new statistics. Effect sizes, confidence intervals, and metaanalysis. London: Routledge.

Cumming, G. (2014). The new statistics: why and how. Psychological Science, 25(1), 7-29. https://doi. org/10.1177/0956797613504966.

Cumming, G., \& Finch, S. (2005). Inference by eye confidence intervals and how to read pictures of data. American Psychologist, 60(2), 170-180. https://doi.org/10.1037/0003-066X.60.2.170.

Delbaere, K., \& Lord, S. (2015). Validation of a trail-making test developed for iPad, 1-4. https://www.neura.edu.au/wp-content/
uploads/2016/05/Trails-iPad_validation.pdf. Accessed: 21.12.2020

Diamond, A. (2013). Executive functions. Annual Review of Psychology, 64, 135-168. https://doi. org/10.1007/978-3-319-42662-4_9.

Diamond, A., \& Ling, D. S. (2016). Conclusions about interventions, programs, and approaches for improving executive functions that appear justified and those that, despite much hype, do not. Developmental Cognitive Neuroscience. https://doi.org/10.1016/j.dcn.2015.11.005.

Erickson, G. B., Citek, K., Cove, M., Wilczek, J., Linster, C., Bjarnason, B., \& Langemo, N. (2011). Reliability of a computer-based system for measuring visual performance skills. Optometry, 82(9), 528-542. https://doi.org/10.1016/j.optm.2011.01.012.

Faubert, J. (2013). Professional athletes have extraordinary skills for rapidly learning complex and neutral dynamic visual scenes. Scientific Reports. https://doi.org/10.1038/srep01154.

Faubert, J., \& Sidebottom, L. (2012). Perceptualcognitive training of athletes. Journal of Clinical Sport Psychology, 6(1), 85-102. https://doi.org/ 10.1123/jcsp.6.1.85.

Fields, R. D. (2015). A new mechanism of nervous system plasticity: activity-dependent myelination. Nature Reviews Neuroscience, 16(12), 756-767. https://doi.org/10.1038/nrn4023.

Fleddermann, M.-T., Heppe, H., \& Zentgraf, K. (2019). Off-court generic perceptual-cognitive training 
in elite volleyball athletes: task-specific effects and levels of transfer. Frontiers in Psychology, 10, 1-12. https://doi.org/10.3389/fpsyg.2019. 01599.

Furley, P., \& Memmert, D. (2011). Studying cognitive adaptations in the field of sport: broad or narrow transfer? A comment on Allen, Fioratou, and McGeorge (2011). Perceptual and Motor Skills, 113(2), 481-488. https://doi.org/10.2466/05.23. PMS.113.5.481-488.

Gilbert, S. J., \& Burgess, P.W. (2008). Executive function. Current Biology, 18(3), R110-R114.

Hadlow, S. M., Panchuk, D., Mann, D. L., Portus, M. R., \& Abernethy, B. (2018). Modified perceptual training in sport: a new classification framework. Journal of Science and Medicine in Sport, 21(9), 950-958. https://doi.org/10.1016/j.jsams.2018. 01.011.

Harris, D.J., Wilson, M.R., \& Vine, S.J. (2018). A systematic review of commercial cognitive training devices: Implications for use in sport. Frontiers in Psychology. https://doi.org/10.3389/ fpsyg.2018.00709.

Homack, S., Lee, D., \& Riccio, C. A. (2005). Test review: Delis-Kaplan executive function system. Journal of Clinical and Experimental Neuropsychology, 27(5), 599-609. https://doi.org/10.1080/ 13803390490918444

Huang, Z., Davis, H., Wolff, A., \& Northoff, G. (2017). Thalamo-sensorimotor functional connectivity correlates with world ranking of olympic, elite, and high performance athletes. Neural Plasticity. https://doi.org/10.1155/2017/1473783.

Huijgen, B. C. H., Leemhuis, S., Kok, N. M., Verburgh, L., Oosterlaan, J., Elferink-Gemser, M. T., \& Visscher, C. (2015). Cognitive functions in elite and subelite youth soccer players aged 13 to 17 years. PLOSONE, 10(12), 1-13. https://doi.org/10.1371/ journal.pone.0144580.

Hüttermann, S., \& Memmert, D. (2017). The attention window: a narrative review of limitations and opportunities influencing the focus of attention. Research Quarterly for Exercise and Sport, 88(2), 169-183. https://doi.org/10.1080/02701367. 2017.1293228.

Hüttermann, S., Memmert, D., \& Simons, D.J. (2014). The size and shape of the attentional "spotlight" varies with differences in sports expertise. Journal of Experimental Psychology: Applied, 20(2), 147-157. https://doi.org/10. 1037/xap0000012.

Ivarsson, A., Andersen, M. B., Johnson, U., \& Lindwall, M (2013). To adjust or not adjust: Nonparametric effect sizes, confidence intervals, and real-world meaning. Psychology of Sport and Exercise, 14(1), 97-102. https://doi.org/10.1016/j.psychsport. 2012.07.007.

Kane, M. J., Hambrick, D. Z., Tuholski, S. W., Wilhelm, O. Payne, T. W., \& Engle, R. W. (2004). The generality of working-memory capacity: a latent-variable approach to verbal and visuospatial memory span and reasoning. Journal of Experimental Psychology: General, 133, 189-217.

Karbach, J., \& Unger, K. (2014). Executive control training from middle childhood to adolescence. Frontiers in Psychology, 5(5), 1-14. https://doi. org/10.3389/fpsyg.2014.00390.

Kreitz, C., Furley, P., Memmert, D., \& Simons, D. J. (2014). Working-memory performance is related to spatial breadth of attention. Psychological Research, 79(6), 1034-1041. https://doi.org/10. 1007/s00426-014-0633-x.

Mangine, G.T., Hoffman, J.R., Wells, A.J., Gonzalez, A. M., Rogowski, J. P., Townsend, J. R., et al. (2014).
Visual tracking speed is related to basketballspecific measures of performance in NBA players. Journal of Strength and Conditioning Research, 28(9), 2406-2414. https://doi.org/10.1519/JSC. 0000000000000550 .

Mann, D.T.Y., Williams, A.M., Ward, P., \& Janelle, C.M. (2007). Perceptual-cognitive expertise in sport: a meta-analysis. Journal of Sport \& Exercise Psychology, 29, 457-478.

Miyake, A., \& Friedman, N.P. (2012). The nature and organization of individual differences in executive functions: four general conclusions. Curren Directions in Psychological Science, 21(1), 8-14. https://doi.org/10.1177/0963721411429458.

Moen, F., Hrozanova, M., \&Stiles, T. (2018). The effects of perceptual-cognitive training with neurotracker on executive brain functions among elite athletes. Cogent Psychology, 5(1), 1-13. https:// doi.org/10.1080/23311908.2018.1544105.

Parsons, B., Magill, T., Boucher, A., Zhang, M., Zogbo, K., Bérubé, S., et al. (2016). Enhancing cognitive function using perceptual-cognitive training. Clinical EEG and Neuroscience, 47(1), 37-47. https://doi.org/10.1177/1550059414563746.

Prakash, R. S., Voss, M. W., Erickson, K. I., \& Kramer, A. F. (2015). Physical activity and cognitive vitality. Annual review of psychology, 66, 769-797.

Roberts, J.W., Strudwick, A. J., \& Bennett, S. J. (2017). Visual function of english premier league socce players. Science and Medicine in Football, 1(2), 178-182. https://doi.org/10.1080/24733938. 2017.1330552.

Romeas, T., Chaumillon, R., Labbé, D., \& Faubert, J. (2019). Combining 3D-MOT with sport decisionmaking for perceptual-cognitive training in virtual reality. Perceptual and Motor Skills, 126(5), 922-948. https://doi.org/10.1177/ 0031512519860286.

Romeas, T., Guldner, A., \& Faubert, J. (2016). 3Dmultiple object tracking training task improves passing decision-making accuracy in soccer players. Psychology of Sport and Exercise, 22, 1-9. https://doi.org/10.1016/j.psychsport.2015.06. 002.

Scharfen, H. E., \& Memmert, D. (2019a). Measurement of cognitive functions in experts and elite athletes: a meta-analytic review. Applied Cognitive Psychology. https://doi.org/10.1002/ acp.3526.

Scharfen, H.E., \& Memmert, D. (2019b). The relationship between cognitive functions and sport-specific motor skills in elite youth soccer players. Frontiers in Psychology, 10, 817. https:// doi.org/10.3389/fpsyg.2019.00817.

Swanson, J. (2005). The Delis-Kaplan executive function system. Canadian Journal of School Psychology, 20(1-2), 117-128. https://doi.org/ 10.1177/0829573506295469.

Sánchez-Cubillo, I., Periáñez, J. A., Adrover-Roig, D., Rodríguez-Sánchez, J. M., Ríos-Lago, M., Tirapu J., \& Barceló, F. (2009). Construct validity of the trail making test: role of task-switching, working memory, inhibition/interference control, and visuomotor abilities. Journal of the International Neuropsychological Society, 15(3), 438-450. https://doi.org/10.1017/S1355617709090626.

Verburgh, L., Scherder, E.J.A., Van Lange, P.A., \& Oosterlaan, J. (2014). Executive functioning in highly talented soccer players. PLOS ONE, 9(3), 91254. https://doi.org/10.1371/journal.pone. 0091254.

Verburgh, L., Scherder, E. J.A., Van Lange, P. A. M., \& Oosterlaan, J. (2016). Do elite and amateur soccer players outperform non-athletes on neurocognitive functioning? A study among 8-12 year old children. PLOS ONE. https://doi. org/10.1371/journal.pone.0165741.

Vestberg, T., Gustafson, R., Maurex, L., Ingvar, M., \& Petrovic, P. (2012). Executive functions predict the success of top-soccer players. PLOS ONE, 7(4), 34731. https://doi.org/10.1371/journal.pone. 0034731.

Vestberg, T., Reinebo, G., Maurex, L., Ingvar, M., \& Petrovic, P. (2017). Core executive functions are associated with success in young elite soccer players. PloS ONE, 12(2), e170845. https://doi. org/10.1371/journal.pone.0170845.

Voss, M. W., Kramer, A. F., Basak, C., Prakash, R. S., \& Roberts, B. (2010). Are expert athletes "expert" in the cognitive laboratory? A meta-analytic review of cognition and sport expertise. Applied Cognitive Psychology, 24(6), 812-826. https:// doi.org/10.1002/acp.1588.

Walton, C.C., Keegan, R. J., Martin, M., \& Hallock, H. (2018). The potential role for cognitive training in sport: more research needed. Frontiers in Psychology, 9, 1121. https://doi.org/10.3389/ fpsyg.2018.01121.

Woods, D. L., Wyma, J. M., Herron, T. J., \& Yund, E.W. (2016). A computerized test of design fluency. PLOS ONE, 11(5), 153952. https://doi.org/10. 1371/journal.pone.0153952.

Yarrow, K., Brown, P., \& Krakauer, J. W. (2009). Inside the brain of an elite athlete: the neural processes that support high achievement in sports. Nature Reviews Neuroscience, 10(8), 585-596. https:// doi.org/10.1038/nrn2672.

Zentgraf, K., Heppe, H., \& Fleddermann, M. T. (2017). Training in interactive sports. A systematic review of practice and transfer effects of perceptual-cognitive training. German Journal of Exercise and Sport Research, 47(1), 2-14. https://doi.org/10.1007/s12662-017-0441-8. 